\title{
Bilattice-Based Squares and Triangles
}

\author{
Ofer Arieli ${ }^{1}$, Chris Cornelis ${ }^{2}$, Glad Deschrijver ${ }^{2}$, and Etienne Kerre ${ }^{2}$ \\ 1 Department of Computer Science, The Academic College of Tel-Aviv, Israel \\ oarieli@mta.ac.il \\ ${ }^{2}$ Fuzziness and Uncertainty Modelling Research Unit, Department of Mathematics \\ and Computer Science, Ghent University, Belgium \\ \{chris.cornelis,glad.deschrijver, etienne.kerre\}@UGent.be
}

\begin{abstract}
In this paper, Ginsberg's/Fitting's theory of bilattices is invoked as a natural accommodation and powerful generalization to both intuitionistic fuzzy sets (IFSs) and interval-valued fuzzy sets (IVFSs), serving on one hand to clarify the exact nature of the relationship between these two common extensions of fuzzy sets, and on the other hand providing a general and intuitively attractive framework for the representation of uncertain and potentially conflicting information.
\end{abstract}

\section{Introduction}

Bilattices are algebraic structures that were introduced by Ginsberg [19] as a general and uniform framework for a diversity of applications in artificial intelligence. In a series of papers it was then shown that these structures may serve as a foundation of many areas, such as logic programming [15], computational linguistics [23], distributed knowledge processing [22], and reasoning with imprecise information $[1,2,18]$. The usefulness of bilattices in the context of fuzzy set theory was recently made explicit in [3], where we demonstrated that socalled bilattice-based 'squares' and 'triangles' provide an elegant framework for bridging between intuitionistic fuzzy sets (IFSs) and interval-valued fuzzy sets (IVFSs), thus also shedding a clear light on the syntactical equivalence of these two commonly encountered extensions of Zadeh's fuzzy sets.

The present work is an elaboration on the latter observation. Starting from a complete lattice, we study the corresponding bilattice-based squares and triangles, compare and relate them to various extensions of IFSs and/or IVFSs that have been proposed in the literature, and equip them with suitable logical connectives. In this sense, this paper can also be viewed as a generalization of other papers [10-12] that refer to particular forms of 'triangle' and 'square', in which the underlying structure is the unit interval.

\section{Preliminaries}

\subsection{Intuitionistic fuzzy sets and interval-valued fuzzy sets}

Intuitionistic fuzzy set (IFS) theory [4] is an extension of fuzzy set theory in which any element $u$ in a universe $U$ is assigned not only a membership degree, 
$\mu_{A}(u)$, but also a non-membership degree $\nu_{A}(u)$, where both degrees are drawn from the unit interval $[0,1]$. While in Zadeh's fuzzy set theory [27] always $\nu_{A}(u)=$ $1-\mu_{A}(u)$, in IFS theory a weaker constraint is imposed: $\nu_{A}(u) \leq 1-\mu_{A}(u){ }^{3}$ IFSs can also be regarded as a particular kind of Goguen's $\mathcal{L}$-fuzzy sets [20], i.e., as mappings from a universe $U$ into the complete lattice $\mathcal{L}^{*}$, defined as follows:

Definition 1. [13] $\mathcal{L}^{*}=\left(L^{*}, \leq_{L^{*}}\right)$, where $L^{*}=\left\{\left(x_{1}, x_{2}\right) \in[0,1]^{2} \mid x_{1}+x_{2} \leq 1\right\}$ and $\left(x_{1}, x_{2}\right) \leq_{L^{*}}\left(y_{1}, y_{2}\right)$ iff $x_{1} \leq y_{1}$ and $x_{2} \geq y_{2}$.

Interval-valued fuzzy set (IVFS) theory is an alternative method of extending fuzzy set theory, motivated by the need to replace crisp, $[0,1]$-valued membership degrees by intervals in $[0,1]$ that approximate the (unknown) membership degrees. Interval-valued fuzzy sets are also $\mathcal{L}$-fuzzy sets, for which the corresponding lattice is $\mathcal{L}^{I}$, defined as follows:

Definition 2. [11] $\mathcal{L}^{I}=\left(L^{I}, \leq_{L^{I}}\right)$, where $L^{I}=\left\{\left[x_{1}, x_{2}\right] \mid\left(x_{1}, x_{2}\right) \in[0,1]^{2}, x_{1} \leq\right.$ $\left.x_{2}\right\}$ and $\left[x_{1}, x_{2}\right] \leq_{L^{I}}\left[y_{1}, y_{2}\right]$ iff $x_{1} \leq y_{1}$ and $x_{2} \leq y_{2}$.

\subsection{Bilattices}

As noted above, bilattices are the mathematical structures used here for relating IFSs and IVFSs. We first review some basic definitions that pertain to bilattices.

Definition 3. [16] A pre-bilattice is a structure $\mathcal{B}=\left(B, \leq_{t}, \leq_{k}\right)$, such that $B$ is a set containing at least two elements, and $\left(B, \leq_{t}\right),\left(B, \leq_{k}\right)$ are complete lattices.

Definition 4. Let $\mathcal{B}=\left(B, \leq_{t}, \leq_{k}\right)$ be a pre-bilattice.

A negation of $\mathcal{B}$ is a unary operation $\neg$ on $B$ satisfying the following properties:
(1) $\neg \neg x=x$
(2) if $x \leq_{t} y$ then $\neg x \geq_{t} \neg y$,
(3) if $x \leq_{k} y$ then $\neg x \leq_{k} \neg y$.

A conflation of $\mathcal{B}$ is a unary operation - on $B$ satisfying the following properties:
(1) $--x=x$
(2) if $x \leq_{k} y$ then $-x \geq_{k}-y$,
(3) if $x \leq_{t} y$ then $-x \leq_{t}-y$.

Definition 5. [19] A bilattice is a structure $\mathcal{B}=\left(B, \leq_{t}, \leq_{k}, \neg\right)$, such that $\left(B, \leq_{t}, \leq_{k}\right)$ is a pre-bilattice with a negation $\neg$.

In the sequel, following the usual notations for the basic bilattice operations, we shall denote by $\wedge$ (respectively, by $\vee$ ) the $\leq_{t}$-meet (the $\leq_{t}$-join) and by $\otimes$ (respectively, by $\oplus$ ) the $\leq_{k}$-meet (the $\leq_{k}$-join) of a bilattice $\mathcal{B}$. $f$ and $t$ denote the $\leq_{t}$-extreme elements, and $\perp, T$ denote the $\leq_{k}$-extreme elements. Intuitively, these elements can be perceived as 'false', 'true', 'unknown' (i.e., neither true nor false) and 'contradictory' (both true and false), respectively. The two partial orders $\leq_{t}$ and $\leq_{k}$ are taken to represent differences in the degree of truth and in the amount of information (respectively), conveyed by a given assertion.

Proposition 1. Let $\mathcal{B}=\left(B, \leq_{t}, \leq_{k}, \neg\right)$ be a bilattice.

\footnotetext{
3 The 'intuitionistic' characterizations of this approach should be understood here in a 'broad' sense, as it loosely denies the law of excluded middle. This approach bears no relationship to the conservative extension of intuitionistic logic [24].
} 
a) [19] For every $x, y$ in $B$ :

$\neg(x \wedge y)=\neg x \vee \neg y, \neg(x \vee y)=\neg x \wedge \neg y, \neg(x \otimes y)=\neg x \otimes \neg y, \neg(x \oplus y)=\neg x \oplus \neg y$. $\neg f=t, \quad \neg t=f, \neg \perp=\perp, \neg \top=\top$.

b) [16] If $\mathcal{B}$ has conflation -, then, for every $x, y$ in $B$ :

$-(x \wedge y)=-x \wedge-y,-(x \vee y)=-x \vee-y,-(x \otimes y)=-x \oplus-y,-(x \oplus y)=-x \otimes-y$.

$-f=f, \quad-t=t, \quad-\perp=\top, \quad-\top=\perp$.

Definition 6. A bilattice $\mathcal{B}=\left(B, \leq_{t}, \leq_{k}, \neg\right)$ is distributive [19] if all the (twelve) possible distributive laws concerning $\wedge, \vee, \otimes$, and $\oplus$ hold.

Following Fitting [14], we consider a special kind of distributive bilattices.

Definition 7. A distributive bilattice $\mathcal{B}=\left(B, \leq_{t}, \leq_{k}, \neg\right)$ with a conflation that commutes with $\neg$ is called classical, if $x \vee-\neg x=t$ for every $x$ in $B$.

\section{Relating IFSs and IVFSs through bilattices}

In this section, we introduce a general context featuring a number of bilatticebased structures to relate and generalize the IFS/IVFS constructs $\mathcal{L}^{*}$ and $\mathcal{L}^{I}$, as well as some of their extensions.

Definition 8. [19] Let $\mathcal{L}=\left(L, \leq_{L}\right)$ be a complete lattice. A (bilattice-based) square is a structure $\mathcal{L}^{2}=\left(L \times L, \leq_{t}, \leq_{k}, \neg\right),{ }^{4}$ where $\neg\left(x_{1}, x_{2}\right)=\left(x_{2}, x_{1}\right)$, and

(1) $\left(x_{1}, x_{2}\right) \leq_{t}\left(y_{1}, y_{2}\right) \Leftrightarrow x_{1} \leq_{L} y_{1}$ and $x_{2} \geq_{L} y_{2}$,

(2) $\left(x_{1}, x_{2}\right) \leq_{k}\left(y_{1}, y_{2}\right) \Leftrightarrow x_{1} \leq_{L} y_{1}$ and $x_{2} \leq_{L} y_{2}$.

An element $\left(x_{1}, x_{2}\right)$ of a square $\mathcal{L}^{2}$ may intuitively be understood such that $x_{1}$ represents the amount of belief for some assertion, and $x_{2}$ is the amount of belief against it. This corresponds to Atanassov's idea [4] of distinguishing between a membership component $\mu_{A}(u)$ and a non-membership component $\nu_{A}(u)$, with the amendment that in the case of a square no restriction like $\mu_{A}(u)+\nu_{A}(u) \leq 1$ for every $u$ in $U$ is imposed. Note also that the $\leq_{t}$-ordering of $\mathcal{L}^{2}$ is completely in line with the partial order of $\mathcal{L}^{*}$; the $\leq_{k}$-ordering additionally discriminates couples in $L^{2}$ according to the amount of information they carry ${ }^{5}$.

Denoting the join and meet operations of the complete lattice $\mathcal{L}$ by $\sqcap$ and $\sqcup$, respectively, we have, for $\left(x_{1}, x_{2}\right),\left(y_{1}, y_{2}\right)$ in $L^{2}$,

$$
\begin{aligned}
& \left(x_{1}, x_{2}\right) \wedge\left(y_{1}, y_{2}\right)=\left(x_{1} \sqcap y_{1}, x_{2} \sqcup y_{2}\right),\left(x_{1}, x_{2}\right) \vee\left(y_{1}, y_{2}\right)=\left(x_{1} \sqcup y_{1}, x_{2} \sqcap y_{2}\right) \\
& \left(x_{1}, x_{2}\right) \otimes\left(y_{1}, y_{2}\right)=\left(x_{1} \sqcap y_{1}, x_{2} \sqcap y_{2}\right),\left(x_{1}, x_{2}\right) \oplus\left(y_{1}, y_{2}\right)=\left(x_{1} \sqcup y_{1}, x_{2} \sqcup y_{2}\right)
\end{aligned}
$$

Moreover, denoting $0_{\mathcal{L}}=\inf L$ and $1_{\mathcal{L}}=\sup L$, it holds that $\perp_{\mathcal{L}^{2}}=\left(0_{\mathcal{L}}, 0_{\mathcal{L}}\right)$, $\top_{\mathcal{L}^{2}}=\left(1_{\mathcal{L}}, 1_{\mathcal{L}}\right), t_{\mathcal{L}^{2}}=\left(1_{\mathcal{L}}, 0_{\mathcal{L}}\right)$, and $f_{\mathcal{L}^{2}}=\left(0_{\mathcal{L}}, 1_{\mathcal{L}}\right)$. When $\mathcal{N}$ is an involution of $\mathcal{L}$, then $-\mathcal{N}\left(x_{1}, x_{2}\right)=\left(\mathcal{N}\left(x_{2}\right), \mathcal{N}\left(x_{1}\right)\right)$ is a conflation of $\mathcal{L}^{2}$.

It is easy to verify that every square $\mathcal{L}^{2}$ is distributive when $\mathcal{L}$ is distributive.

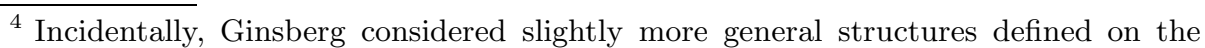
cartesian product of two not necessarily equal complete lattices.

${ }^{5}$ Note also that the $\leq_{k}$-order of a square appears to correspond to the partial order of $\mathcal{L}^{I}$.
} 
Example 1. Let $\mathcal{L}_{2}=(\{0,1\}, \leq)$ and $\mathcal{L}_{3}=\left(\left\{0, \frac{1}{2}, 1\right\}, \leq\right)$, with $\leq$ in each case the usual ordering. The bilattices $\mathcal{L}_{2}^{2}$ and $\mathcal{L}_{3}^{2}$ are shown in Figure 1 . In the literature, these structures are commonly referred to as $\mathcal{F} \mathcal{O U} \mathcal{R}$ (after Belnap's [6,7] original four-valued logic) and $\mathcal{N} \mathcal{I N \mathcal { E }}$ (see, e.g., [1, 2]), respectively. Both these bilattices are distributive, and $\mathcal{F O U R}$ is also classical, while $\mathcal{N} \mathcal{I N E}$ is not. An example of a square with an infinite amount of elements is, for instance, $([0,1], \leq)^{2}$.
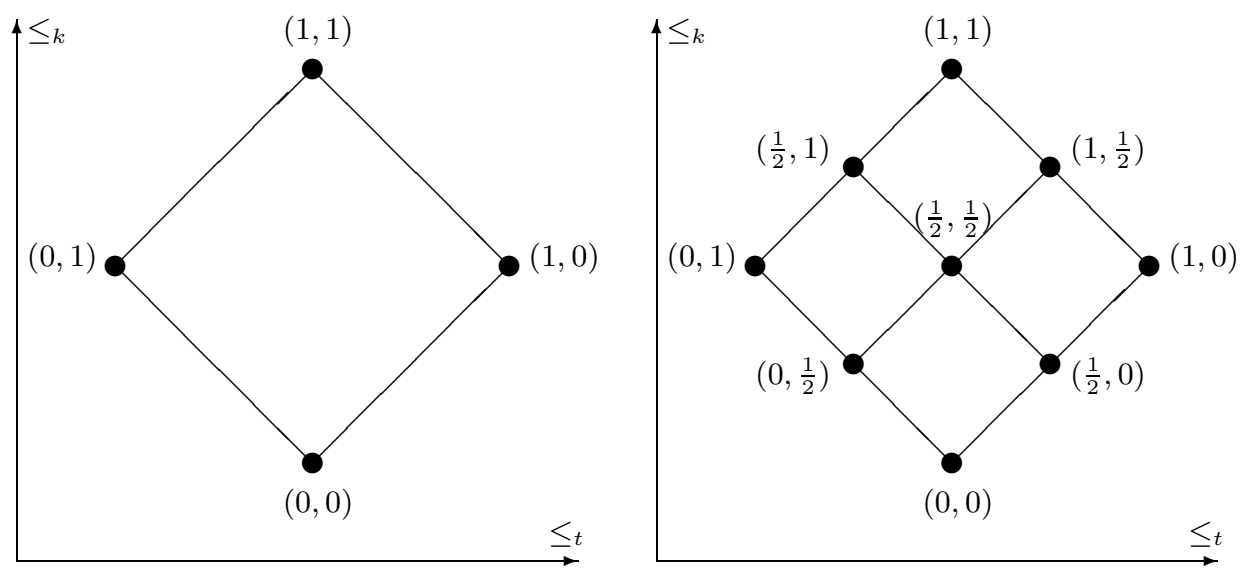

Fig. 1. The bilattices $\mathcal{L}_{2}^{2}$ and $\mathcal{L}_{3}^{2}$

The second bilattice-based structure investigated here is due to Fitting [16].

Definition 9. Let $\mathcal{L}=\left(L, \leq_{L}\right)$ be a complete lattice, and $I(L)=\left\{\left[x_{1}, x_{2}\right] \mid\right.$ $\left.\left(x_{1}, x_{2}\right) \in L^{2}, x_{1} \leq_{L} x_{2}\right\}$. A (bilattice-based) triangle is a structure $\mathcal{I}(\mathcal{L})=$ $\left(I(L), \leq_{t}, \leq_{k}\right)$, where

(1) $\left[x_{1}, x_{2}\right] \leq_{t}\left[y_{1}, y_{2}\right] \Leftrightarrow x_{1} \leq_{L} y_{1}$ and $x_{2} \leq_{L} y_{2}$,

(2) $\left[x_{1}, x_{2}\right] \leq_{k}\left[y_{1}, y_{2}\right] \Leftrightarrow x_{1} \leq_{L} y_{1}$ and $x_{2} \geq_{L} y_{2}$.

Note that a triangle $\mathcal{I}(\mathcal{L})$ is in fact not a (pre-)bilattice, since the substructure $\left(I(L), \leq_{k}\right)$ is not a lattice (the supremum of any two elements does not necessarily exist). Still, triangles are very much in the same spirit as bilattices, since the $\leq_{k}$-ordering also represents differences in the amount of information that each interval exhibits.

Example 2. The triangles $\mathcal{I}\left(\mathcal{L}_{2}\right)$ and $\mathcal{I}\left(\mathcal{L}_{3}\right)$ are shown in Figure 2. When $\mathcal{L}$ is the unit interval with the usual ordering, $\mathcal{I}(\mathcal{L})$ is a structure that extends the lattice $\mathcal{L}^{I}$ in the sense that $\mathcal{L}^{I}$ is exactly $\left(I([0,1]), \leq_{t}\right)$. Moreover, $\mathcal{I}(\mathcal{L})$ also contains the partially ordered set $\left(I([0,1]), \leq_{k}\right)$ that orders intervals according to their exactness.

Definition 10. [16] Let $\mathcal{B}=\left(B, \leq_{t}, \leq_{k}, \neg\right)$ be a bilattice with a conflation -. An element $x$ in $B$ is called exact with respect to this conflation if $x=-x$; it is consistent if $x \leq_{k}-x$. 

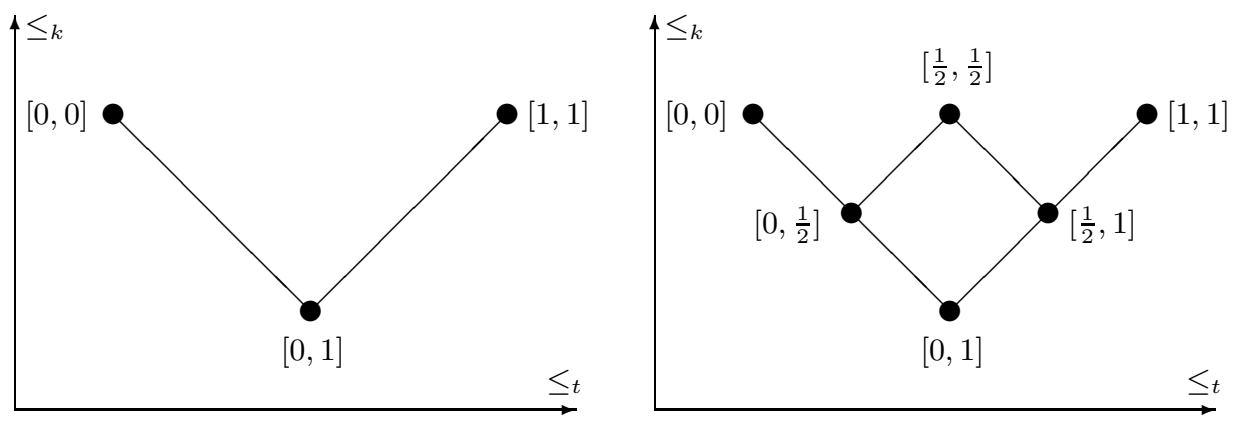

Fig. 2. The triangles $\mathcal{I}\left(\mathcal{L}_{2}\right)$ and $\mathcal{I}\left(\mathcal{L}_{3}\right)$

Intuitively, exact elements exhibit precise information, while the consistent ones endorse non-contradictory evidence about their assertions.

Definition 11. Let - be a conflation of a bilattice $\mathcal{B}=\left(B, \leq_{t}, \leq_{k}, \neg\right)$. Denote by $\mathcal{C}^{-}(\mathcal{B})$ the substructure of $\mathcal{B}$ with the consistent elements (w.r.t. - ) of $B$.

The following proposition relates squares and triangles:

Proposition 2. [16] Suppose that $\mathcal{L}$ is a complete lattice with an involution $\mathcal{N}$. Then $\mathcal{I}(\mathcal{L})$ is isomorphic to $\mathcal{C}^{-\mathcal{N}}\left(\mathcal{L}^{2}\right)$.

The isomorphism $f$ between $\mathcal{I}(\mathcal{L})$ and $\mathcal{C}^{-\mathcal{N}}\left(\mathcal{L}^{2}\right)$ for Proposition 2 is given by $f\left(\left[x_{1}, x_{2}\right]\right)=\left(x_{1}, \mathcal{N}\left(x_{2}\right)\right)$. If $L$ is the unit interval, $f\left(\left[x_{1}, x_{2}\right]\right)=\left(x_{1}, 1-x_{2}\right)$, which is the transformation considered in [10] for switching between IVFSs and IFSs. The above result shows that the same transformation is useful not only for relating $\mathcal{L}^{I}$ and $\mathcal{L}^{*}$ (i.e., when the underlying lattice is the unit interval), but also for any complete lattice with an involution.

The result above may also serve as a clarification of Atanassov's decision to consider only the elements $\left(x_{1}, x_{2}\right)$ in $[0,1]^{2}$ s.t. $x_{1}+x_{2} \leq 1$ : these are exactly the consistent elements of $[0,1]^{2}$, when the conflation is defined in $[0,1]^{2}$, by $-\left(x_{1}, x_{2}\right)=\left(1-x_{2}, 1-x_{1}\right)$. The fact that we consider super-lattices of $\mathcal{L}^{*}$ (i.e., all the elements in $[0,1]^{2}$ are considered) allows us to introduce elements such as $\left(x_{1}, x_{2}\right)=(1,1)$, in which the membership degree $\left(x_{1}\right)$ and the non-membership degree $\left(x_{2}\right)$ are both maximal, so we have a totally inconsistent belief in this case. As an important aspect of fuzzy set theory is reasoning with uncertainty, such values should not be ruled out.

Note 1. In [5], Atanassov introduced a pair of bijections between $L^{*}$ and $[0,1]^{2}$, which at first glance seems to shatter the remarks made above that the latter is a more expressive structure. It was shown in [11], however, that these bijections do not preserve order and hence they do not constitute an isomorphism between $\mathcal{L}^{*}$ and $\left([0,1]^{2}, \leq_{t}\right)$. The following proposition generalizes this result to any complete lattice $\mathcal{L}$ with an involution $\mathcal{N}$.

Proposition 3. For a complete lattice $\mathcal{L}=\left(L, \leq_{L}\right)$ with an involution $\mathcal{N}$, the structures $\mathcal{L}^{2}$ and $\mathcal{C}^{-\mathcal{N}}\left(\mathcal{L}^{2}\right)$ are not isomorphic. 


\section{Graded (bilattice-based) logical connectives}

In this section we recall some common extensions to $\mathcal{L}$-fuzzy set theory of the main connectives of classical logic, and show how they can be related to bilattices. In what follows $\mathcal{L}=\left(L, \leq_{L}\right)$ is a complete lattice, $0_{\mathcal{L}}=\inf L$ and $1_{\mathcal{L}}=\sup L$.

\subsection{Negation}

Definition 12. A negator on $\mathcal{L}$ is any $\leq_{L}$-decreasing mapping $\mathcal{N}: L \rightarrow L$ satisfying $\mathcal{N}\left(0_{\mathcal{L}}\right)=1_{\mathcal{L}}$ and $\mathcal{N}\left(1_{\mathcal{L}}\right)=0_{\mathcal{L}}$. If, for every $x$ in $L, \mathcal{N}(\mathcal{N}(x))=x$, then $\mathcal{N}$ is called an involutive negator on $\mathcal{L}$.

The operation $\neg$ in Definition 5 is an involutive negator on the lattice $\left(B, \leq_{t}\right)$. Therefore, the operation $\neg$, defined on the square $\mathcal{L}^{2}$ by $\neg\left(x_{1}, x_{2}\right)=\left(x_{2}, x_{1}\right)$, is an involutive negator on $\left(L^{2}, \leq_{t}\right)$. If a bilattice $\mathcal{B}$ has a conflation - , then by its definition this operation is an involutive negator on the lattice $\left(B, \leq_{k}\right)$.

Suppose now that $\mathcal{N}$ is an involutive negator on $\mathcal{L}$. Then, as we have shown above, a conflation $-_{\mathcal{N}}$ of $\mathcal{L}^{2}$ may be defined by $-_{\mathcal{N}}\left(x_{1}, x_{2}\right)=\left(\mathcal{N}\left(x_{2}\right), \mathcal{N}\left(x_{1}\right)\right)$. In this case, another natural negator $\sim_{\mathcal{N}}$ on $\left(L^{2}, \leq_{t}\right)$ is obtained by combining $\neg$ and $-_{\mathcal{N}}$ as follows: $\sim_{\mathcal{N}}\left(x_{1}, x_{2}\right)=\neg-\mathcal{N}\left(x_{1}, x_{2}\right)=\left(\mathcal{N}\left(x_{1}\right), \mathcal{N}\left(x_{2}\right)\right)$.

One might wonder if there exist other 'interesting' negators apart from the prototypical ones described above. In [12] it was shown however that for the particular structure $\left([0,1]^{2}, \leq_{t}\right)$ all involutive negators can be generated by simple transformations of the two basic choices $\neg$ and $\sim_{\mathcal{N}}$. The next proposition is a generalization of that result to squares.

Definition 13. For $x=\left(x_{1}, x_{2}\right)$ in $L^{2}$, denote: $p r_{1}(x)=x_{1}$ and $p r_{2}(x)=x_{2}$.

Proposition 4. Let $\mathcal{L}=\left(L, \leq_{L}\right)$ be a chain. An operation $\mathfrak{N}$ is an involutive negator on $\left(L^{2}, \leq_{t}\right)$ iff either

$$
\mathfrak{N}\left(x_{1}, x_{2}\right)=\left(\mathcal{N}_{1}\left(x_{1}\right), \mathcal{N}_{2}\left(x_{2}\right)\right)
$$

where $\mathcal{N}_{1}$ and $\mathcal{N}_{2}$ are two involutive negators on $\mathcal{L}$ such that $\mathcal{N}_{1}(x)=p r_{1} \mathfrak{N}\left(x, 0_{\mathcal{L}}\right)$ and $\mathcal{N}_{2}(x)=p r_{2} \mathfrak{N}\left(0_{\mathcal{L}}, x\right)$, or

$$
\mathfrak{N}\left(x_{1}, x_{2}\right)=\left(\varphi\left(x_{2}\right), \varphi^{-1}\left(x_{1}\right)\right)
$$

where $\varphi$ is an increasing permutation of $\mathcal{L}$ such that $\varphi(x)=p r_{1} \mathfrak{N}\left(0_{\mathcal{L}}, x\right)$.

Clearly, $\sim_{\mathcal{N}}$ is obtained from Expression (1) where $\mathcal{N}=\mathcal{N}_{1}=\mathcal{N}_{2}$, and $\neg$ is obtained from Expression (2) where $\varphi$ is the identity permutation of $L$.

One of the advantages of $\sim_{\mathcal{N}}$ is that it preserves the following weakened version of the law of the excluded middle.

Definition 14. An involutive negator $\mathcal{N}$ on $\mathcal{L}$ is called Kleene negator, if for all $x, y$ in $L, x \wedge_{L} \mathcal{N}(x) \leq_{L} y \vee_{L} \mathcal{N}(y)$. 
The intuition here is that even when the excluded middle or contradiction law do not hold, 'intended' contradictions should not surpass 'intended' tautologies.

Proposition 5. If $\mathcal{N}$ is a Kleene negator on $\mathcal{L}$, then $\sim_{\mathcal{N}}$ is a Kleene negator on $\left(L^{2}, \leq_{t}\right)$.

Unlike $\sim_{\mathcal{N}}$, the negator $\neg$ never satisfies Kleene's condition (to see this, consider, for instance, $\left(x_{1}, x_{2}\right)=\left(1_{\mathcal{L}}, 1_{\mathcal{L}}\right)$ and $\left.\left(y_{1}, y_{2}\right)=\left(0_{\mathcal{L}}, 0_{\mathcal{L}}\right)\right)$. On the other hand, $\sim_{\mathcal{N}}$ also has some disadvantages. For instance, it cannot serve as a (bilattice) negation on $\mathcal{L}^{2}$ in the sense of Definition 5 , since it does not preserve the $\leq_{k^{-}}$ order (In $\mathcal{L}_{2}^{2}$, for example, although $(1,0) \leq_{k}(1,1)$, still $\sim_{\mathcal{N}}(1,0) \not_{k} \sim_{\mathcal{N}}(1,1)$ ).

Consider now negators in triangles $\mathcal{I}(\mathcal{L})$, or — equivalently — the substructure $\mathcal{C}^{-\mathcal{N}}(\mathcal{L})$. By the following proposition, it is rather straightforward to find an analogous definition of $\neg$ for these structures, while for $\sim_{\mathcal{N}}$ this is not possible.

Proposition 6. Let $\mathcal{L}$ be a complete lattice with an involutive negator $\mathcal{N}$. Then $\mathcal{C}^{-\mathcal{N}}(\mathcal{L})$ is closed under $\neg$ but not under $\sim_{\mathcal{N}}$.

Thus, for the negator $\neg$, a corresponding triangle operation may be obtained by applying the isomorphism $f\left(\left[x_{1}, x_{2}\right]\right)=\left(x_{1}, \mathcal{N}\left(x_{2}\right)\right)$, used in the context of Proposition 2, to obtain an operation $\mathfrak{N}$ defined, for every $\left[x_{1}, x_{2}\right]$ in $I(L)$, by

$$
\mathfrak{N}\left(\left[x_{1}, x_{2}\right]\right)=\left[\mathcal{N}\left(x_{2}\right), \mathcal{N}\left(x_{1}\right)\right]
$$

As $\left[\mathcal{N}\left(x_{2}\right), \mathcal{N}\left(x_{1}\right)\right]$ is an interval, $\mathfrak{N}$ is an involutive negator on $\left(I(L), \leq_{t}\right)$.

Next we show, as we did for squares (cf. Proposition 4), that Expression (3) is a characterization of involutive negators in many common triangles:

Definition 15. For $x=\left[x_{1}, x_{2}\right] \in I(L)$, denote: $l(x)=x_{1}$ and $r(x)=x_{2}$.

Proposition 7. Let $\mathcal{L}=\left(L, \leq_{L}\right)$ be a chain with at least four elements. An operation $\mathfrak{N}$ is an involutive negator on $\left(I(L), \leq_{t}\right)$ iff $\mathfrak{N}\left(\left[x_{1}, x_{2}\right]\right)=\left[\mathcal{N}\left(x_{2}\right), \mathcal{N}\left(x_{1}\right)\right]$, where $\mathcal{N}$ is an involutive negator on $\mathcal{L}$ such that $\mathcal{N}(x)=r\left(\mathfrak{N}\left[x, 1_{\mathcal{L}}\right]\right)=l\left(\mathfrak{N}\left[0_{\mathcal{L}}, x\right]\right)$.

Proposition 7 is not true unless the chain $\mathcal{L}$ has at least four elements:

Example 3. Consider a mapping $\mathcal{N}$ on $\left(I\left(L_{3}\right), \leq_{t}\right)$, defined as follows:

$$
\mathcal{N}\left(\left[x_{1}, x_{2}\right]\right)= \begin{cases}{\left[\frac{1}{2}, \frac{1}{2}\right]} & \text { if }\left[x_{1}, x_{2}\right]=[0,1] \\ {[0,1]} & \text { if }\left[x_{1}, x_{2}\right]=\left[\frac{1}{2}, \frac{1}{2}\right] \\ {\left[1-x_{2}, 1-x_{1}\right]} & \text { otherwise }\end{cases}
$$

It is easy to check that this is an involutive negator on $\left(I\left(L_{3}\right), \leq_{t}\right)$, which is not of the form of Expression (3) (thus it is not generated as described in Proposition 7).

In $[12]$ it is shown that there does not exist a Kleene negator on $\mathcal{I}\left([0,1], \leq_{t}\right)$. The following example shows that this does not hold in general for any triangle. 
Example 4.

a) The operation $\mathcal{N}$, defined by $\mathcal{N}([0,0])=[1,1], \mathcal{N}([1,1])=[0,0]$ and $\mathcal{N}([0,1])=$ $[0,1]$ is a Kleene negator on $\left(I\left(L_{2}\right), \leq_{t}\right)$.

b) The mapping $\mathcal{N}$ of Example 3 is a Kleene negator on $\left(I\left(L_{3}\right), \leq_{t}\right)$.

Proposition 8. Let $\mathcal{L}=\left(L, \leq_{L}\right)$ be a chain with at least four elements. Then there does not exist a Kleene negator on $\left(I(L), \leq_{t}\right)$.

\subsection{Conjunction and disjunction}

Definition 16. A triangular norm (a t-norm, for short) on $\mathcal{L}=\left(L, \leq_{L}\right)$ is a mapping $\mathcal{T}: L \times L \rightarrow L$ that is $\leq_{L}$-increasing in both arguments, commutative, associative, and satisfies, for every $x$ in $L, \mathcal{T}\left(1_{\mathcal{L}}, x\right)=x$.

Definition 17. A triangular conorm (a t-conorm, for short) on $\mathcal{L}=\left(L, \leq_{L}\right)$ is a mapping $\mathcal{S}: L \times L \rightarrow L$ that is $\leq_{L}$-increasing in both arguments, commutative, associative, and satisfies, for every $x$ in $L, \mathcal{S}\left(0_{\mathcal{L}}, x\right)=x$.

Given a pre-bilattice $\mathcal{B}=\left(B, \leq_{t}, \leq_{k}\right)$, its $\leq_{t}$-meet $\wedge$ and $\leq_{k}$-meet $\otimes$ are clearly t-norms on $\left(B, \leq_{t}\right)$ and $\left(B, \leq_{k}\right)$, respectively. Also, the $\leq_{t}$-join $\vee$ and the $\leq_{k}$-join $\oplus$ of $\mathcal{B}$ are t-conorms on $\left(B, \leq_{t}\right)$, and $\left(B, \leq_{k}\right)$, respectively. This implies that for a complete lattice $\mathcal{L}=(L, \leq)$ with a meet $\wedge_{L}$ and a join $\vee_{L}, \mathcal{T}_{\leq_{t}}\left(\left(x_{1}, x_{2}\right),\left(y_{1}, y_{2}\right)\right)=\left(x_{1} \wedge_{L} y_{1}, x_{2} \vee_{L} y_{2}\right)$ is a t-norm on $\left(L^{2}, \leq_{t}\right)$ and $\mathcal{T}_{\leq_{k}}\left(\left(x_{1}, x_{2}\right),\left(y_{1}, y_{2}\right)\right)=\left(x_{1} \wedge_{L} y_{1}, x_{2} \wedge_{L} y_{2}\right)$ is a t-norm on $\left(L^{2}, \leq_{k}\right)$. Similarly, $\mathcal{S}_{\leq_{t}}\left(\left(x_{1}, x_{2}\right),\left(y_{1}, y_{2}\right)\right)=\left(x_{1} \vee_{L} y_{1}, x_{2} \wedge_{L} y_{2}\right)$ is a t-conorm on $\left(L^{2}, \leq_{t}\right)$ and $\mathcal{S}_{\leq_{k}}\left(\left(x_{1}, x_{2}\right),\left(y_{1}, y_{2}\right)\right)=\left(x_{1} \vee_{L} y_{1}, x_{2} \vee_{L} y_{2}\right)$ is a t-conorm on $\left(L^{2}, \leq_{k}\right)$. Also, $\mathcal{T}_{\leq_{t}}$ is the $\leq_{t}$-greatest t-norm of $\left(L^{2}, \leq_{t}\right)$ and $\mathcal{T}_{\leq_{k}}$ is the $\leq_{k}$-greatest t-norm of $\left(L^{2}, \leq_{k}\right)$. Similarly, $\mathcal{S}_{\leq_{t}}$ and $\mathcal{S}_{\leq_{k}}$ are, respectively, the $\leq_{t}$-smallest t-conorm of $\left(L^{2}, \leq_{t}\right)$ and the $\leq_{k}$-smallest t-conorm of $\left(L^{2}, \leq_{k}\right)$.

The definition of $\mathcal{T}_{\leq_{t}}, \mathcal{S}_{\leq_{t}}, \mathcal{T}_{\leq_{k}}$, and $\mathcal{S}_{\leq_{k}}$ is an example of an effective way of generating t-(co)norms on (substructures of) squares $\mathcal{L}^{2}$ by taking advantage of existing connectives on the underlying lattice $\mathcal{L}$. This leads us to define the notion of $\mathcal{L}$-representability.

Definition 18. Let $\mathcal{L}=\left(L, \leq_{L}\right)$ be a complete lattice. A t-norm $\mathfrak{T}$ on $\left(L^{2}, \leq_{t}\right)$ (respectively, a t-conorm $\mathfrak{S}$ ) is called $\mathcal{L}$-representable, if there exist a t-norm $\mathcal{T}$ and a t-conorm $\mathcal{S}$ on $\mathcal{L}$ (respectively, a t-conorm $\mathcal{S}^{\prime}$ and a t-norm $\mathcal{T}^{\prime}$ on $\mathcal{L}$ ) such that, for every $\left(x_{1}, x_{2}\right),\left(y_{1}, y_{2}\right)$ in $L^{2}$,

$$
\begin{aligned}
& \mathfrak{T}\left(\left(x_{1}, x_{2}\right),\left(y_{1}, y_{2}\right)\right)=\left(\mathcal{T}\left(x_{1}, y_{1}\right), \mathcal{S}\left(x_{2}, y_{2}\right)\right) \\
& \mathfrak{S}\left(\left(x_{1}, x_{2}\right),\left(y_{1}, y_{2}\right)\right)=\left(\mathcal{S}^{\prime}\left(x_{1}, y_{1}\right), \mathcal{T}^{\prime}\left(x_{2}, y_{2}\right)\right)
\end{aligned}
$$

$\mathcal{T}$ and $\mathcal{S}$ (resp. $\mathcal{S}^{\prime}$ and $\mathcal{T}^{\prime}$ ) are called the representants of $\mathfrak{T}$ (resp. $\mathfrak{S}$ ).

Analogously, $\mathcal{L}$-representable t- $\left(\right.$ co)norms on $\left(L^{2}, \leq_{k}\right)$ can be defined in the obvious way. 
The definition above allows a straightforward construction of t-(co)norms by operations that meet Definitions 16 and 17; it suffices to take any t-norm $\mathcal{T}$ and t-conorm $\mathcal{S}$ on $\mathcal{L}$, and to use them as representants in formulas (4) and (5) above. The converse, however, is not true; not any t-(co)norm on $\mathcal{L}^{2}$ can be obtained by a representation. For instance, in [21] it is shown that the mapping $\mathcal{T}:[0,1]^{2} \rightarrow[0,1]$, given by:

$$
\mathcal{T}\left(\left(x_{1}, x_{2}\right),\left(y_{1}, y_{2}\right)\right)=\left(\min \left(x_{1}, y_{1}, \max \left(x_{2}, y_{2}\right)\right), \min \left(x_{2}, y_{2}\right)\right)
$$

is indeed a t-norm on $\left([0,1]^{2}, \leq_{k}\right)$, but clearly it is not $\mathcal{L}$-representable, since its first component also depends on $x_{2}$ and $y_{2}$.

Next we relate t-norms and t-conorms by appropriate negators. A natural way of doing so is to impose de Morgan's laws.

Definition 19. Let $\mathcal{T}$ be a t-norm on $\mathcal{L}$, and let $\mathcal{N}$ be an involutive negator on $\mathcal{L}$. A t-conorm $\mathcal{S}$ on $\mathcal{L}$ defined by $\mathcal{S}(x, y)=\mathcal{N}(\mathcal{T}(\mathcal{N}(x), \mathcal{N}(y)))$ is called the $\mathcal{N}$-dual of $\mathcal{T}$. A t-norm on $\mathcal{L}$ that is the $\mathcal{N}$-dual of a given t-conorm, is defined on $\mathcal{L}$ in a similar way.

For $\mathcal{L}$-representable t-norms with $\mathcal{N}$-dual representants on e.g. $\left(L^{2}, \leq_{t}\right)$, the choice of the negator $\sim_{\mathcal{N}}$ or $\neg$ does not affect the identity of the dual t-conorm.

Proposition 9. Suppose that $\mathfrak{T}$ is an $\mathcal{L}$-representable $t$-norm on $\left(L^{2}, \leq_{t}\right)$ with representants $(\mathcal{T}, \mathcal{S})$, such that $\mathcal{T}$ is the $\mathcal{N}$-dual t-norm of $\mathcal{S}$ and $\mathcal{N}$ is an involutive negator on $\mathcal{L}$. Then the $\left(\sim_{\mathcal{N}}\right)$-dual and the $(\neg)$-dual of $\mathfrak{T}$ are the same.

A similar discussion applies also to the definitions of t-(co)norms on (substructures of $\mathcal{I}(\mathcal{L})$, with the caveat that $(1)$ there are no t-norms and t-conorms on $\left(I(L), \leq_{k}\right)$, and $(2)$ in the choice of representants on $\mathcal{L}$ it must be assured that the resulting composite operation always yields an element of $I(L)$.

\subsection{Implication}

Definition 20. An implicator on a lattice $\mathcal{L}$ is a mapping $\mathcal{I}: L \times L \rightarrow L, \leq_{L^{-}}$ increasing in its first component and $\leq_{L}$-decreasing in its second component, such that $\mathcal{I}\left(0_{\mathcal{L}}, 0_{\mathcal{L}}\right)=1_{\mathcal{L}}, \mathcal{I}\left(1_{\mathcal{L}}, 0_{\mathcal{L}}\right)=0_{\mathcal{L}}, \mathcal{I}\left(0_{\mathcal{L}}, 1_{\mathcal{L}}\right)=1_{\mathcal{L}}$, and $\mathcal{I}\left(1_{\mathcal{L}}, 1_{\mathcal{L}}\right)=1_{\mathcal{L}}$.

Given a t-norm $\mathcal{T}$ and an implicator $\mathcal{I}$ on $\mathcal{L}$, it is usual to require the following condition, known as the residuation principle.

$$
\mathcal{T}(x, z) \leq_{L} y \Leftrightarrow z \leq_{L} \mathcal{I}(x, y)
$$

This leads to the following class of implicators:

Definition 21. Let $\mathcal{T}$ be a t-norm on $\mathcal{L}$. An $\mathcal{R}$-implicator $\mathcal{I}_{\mathcal{T}}$ (the residuum of $\mathcal{T})$ is defined, for every $x, y$ in $L$, by $\mathcal{I}_{\mathcal{T}}(x, y)=\sup \left\{z \in L \mid \mathcal{T}(x, z) \leq_{L} y\right\}$.

Another definition of a family of implicators is motivated by the classical definition of the material implication $x \rightarrow y$ as $\neg x \vee y$. 
Definition 22. Let $\mathcal{S}$ be a t-conorm and $\mathcal{N}$ a negator on $\mathcal{L}$. The $\mathcal{S}$-implicator $\mathcal{I}_{\mathcal{S}, \mathcal{N}}\left(\right.$ generated by $\mathcal{S}$ and $\mathcal{N}$ ) is defined by $\mathcal{I}_{\mathcal{S}, \mathcal{N}}(x, y)=\mathcal{S}(\mathcal{N}(x), y)$.

Clearly, each $\mathcal{R}$-implicator and $\mathcal{S}$-implicator is in particular an implicator. Moreover, these definitions reveal that very often implicators are linked to 'simpler' connectives. Also, we can exploit the classical equivalence between the formulas $x \rightarrow f$ and $\neg x$, to define the following special kind of negator on $\mathcal{L}$.

Definition 23. Let $\mathcal{L}=(L, \leq)$ be a complete lattice with an implicator $\mathcal{I}$. Then $\mathcal{N}_{\mathcal{I}}$ defined by $\mathcal{N}_{\mathcal{I}}(x)=\mathcal{I}\left(x, 0_{\mathcal{L}}\right)$, is called the induced negator of $\mathcal{I}$.

Examples of all the above operations on bilattice-based squares and triangles are thus easy to generate using the constructs introduced in the previous sections.

Proposition 10. Let $\mathcal{B}=\left(B, \leq_{t}, \leq_{k}, \neg\right)$ be a classical bilattice with conflation -. Then $\mathcal{I}_{\wedge}=\mathcal{I}_{(\vee, \sim)}$, thus $\mathcal{I}_{\wedge}$ is an $\mathcal{S}$-implicator and an $\mathcal{R}$-implicator on $\left(B, \leq_{t}\right)$.

Proposition 10 substantiates the claim, hinted at by Definition 7 , that in classical bilattices the combination ' $-\neg$ ' is the one that really plays the role of Boolean negation, and $x \vee-\neg x$ are the analogies of classical tautologies.

Definition 24. Let $\mathcal{L}=\left(L, \leq_{L}\right)$ be a complete lattice. Let $\mathcal{T}$ be a t-norm on $\mathcal{L}$ for which Equation (7) holds together with $\mathcal{I}=\mathcal{I}_{\mathcal{T}}$, and for every $x, y$ in $L$,

$$
\mathcal{I}_{\mathcal{T}}\left(\mathcal{I}_{\mathcal{T}}(x, y), y\right)=\mathcal{I}_{\mathcal{T}}\left(\mathcal{I}_{\mathcal{T}}(y, x), x\right)=x \vee_{L} y .
$$

Then $\left(L, \leq_{L}, \mathcal{T}\right)$ is called an $M V$-algebra. ${ }^{6}$

Proposition 10 follows from the following observation and the facts that if $\left(B, \leq_{t}, \leq_{k}, \neg\right)$ is a classical bilattice then $(B, \wedge, \vee,-\neg)$ is a Boolean algebra, and any Boolean algebra $\left(B, \wedge_{B}, \vee_{B}, \neg_{B}\right)$ is an MV-algebra, where $\mathcal{T}=\wedge_{B}$ (see [26]).

Proposition 11. In an MV-algebra $\left(L, \leq_{L}, \mathcal{T}\right)$, the mappings $\mathcal{I}_{\mathcal{T}}$ and $\mathcal{I}_{\mathcal{S}, \mathcal{N}}$, where $\mathcal{N}=\mathcal{N}_{\mathcal{I}_{\mathcal{T}}}$ and $\mathcal{S}$ is the $\mathcal{N}$-dual of $\mathcal{T}$, are identical.

Now we investigate what happens in squares that correspond to non-classical bilattices. The following proposition presents the general picture.

Proposition 12. Let $\mathcal{L}=\left(L, \leq_{L}\right)$ be a complete lattice, and let $\mathfrak{T}$ be a t-norm on $\left(L^{2}, \leq_{t}\right)$. Then $\left(L^{2}, \leq_{t}, \mathfrak{T}\right)$ is an MV-algebra if and only if there exist t-norms $\mathcal{T}$ and $\mathcal{T}^{\prime}$ on $\mathcal{L}$ such that $\left(L, \leq_{L}, \mathcal{T}\right)$ and $\left(L, \leq_{L}, \mathcal{T}^{\prime}\right)$ are both MV-algebras, and such that $\mathfrak{T}$ is $\mathcal{L}$-representable with representants $\mathcal{T}$ and $\mathcal{S}$, where $\mathcal{S}$ is the $\mathcal{N}$-dual t-conorm of a t-norm $\mathcal{T}^{\prime}$ for some involutive negator $\mathcal{N}$.

We turn now to triangles. In these structures the situation is complicated by the fact that there need not exist a Kleene negator on $\left(I(L), \leq_{t}\right)$, while this is a prerequisite of an $\mathrm{MV}$-algebra [9]. ${ }^{7}$ The following example summarizes previous findings when $\mathcal{L}$ is the unit interval.

\footnotetext{
${ }^{6}$ This definition is not a reproduction of the original, lengthy one, given in [8], but is rather a minimal characterization in terms of required properties (see e.g. [26]).

${ }^{7}$ Indeed, if $\left(\mathcal{L}, \leq_{L}, \mathcal{T}\right)$ is an MV-algebra, then $\mathcal{N}_{\mathcal{I}_{\mathcal{T}}}$ is a Kleene negator on $\mathcal{L}$; see $[9$, Theorem 2.31].
} 
Example 5. Consider the lattice $\left(I([0,1]), \leq_{t}\right)$. The mapping $\mathcal{T}\left(\left[x_{1}, x_{2}\right],\left[y_{1}, y_{2}\right]\right)=$ $\left[\max \left(0, x_{1}+y_{1}-1\right), \max \left(0, x_{2}-1+y_{1}, y_{2}-1+x_{1}\right)\right]$ is a non-representable t-norm and it satisfies the residuation principle (7) together with $\mathcal{I}=\mathcal{I}_{\mathcal{T}}$. Moreover,

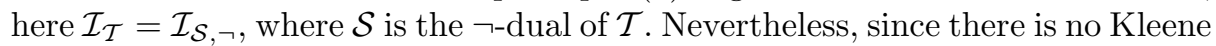
negator on $\left(I([0,1]), \leq_{t}\right)$, the triple $\left(I([0,1]), \leq_{t}, \mathcal{T}\right)$ is not an MV-algebra.

Example 5 thus shows that the property of having coinciding $\mathcal{R}$ - and $\mathcal{S}$ implicators is not unique to MV-algebras. Conversely, one might also wonder if substructures of bilattice-based triangles can ever be MV-algebras; the following example answers this question in the affirmative.

Example 6. Consider the triangle $\mathcal{I}\left(\mathcal{L}_{2}\right)$ from Example 2. As we have seen, the mapping $\mathcal{N}$ defined in Example 4 is a Kleene negator on $\left(I\left(L_{2}\right), \leq_{t}\right)$. Consider the following truth tables that define a t-norm $\mathcal{T}$ and an implicator $\mathcal{I}$ on $\left(I\left(L_{2}\right), \leq_{t}\right)$ :

\begin{tabular}{c|cccc|ccc}
$\mathcal{T}$ & {$[0,0]$} & {$[1,1]$} & {$[0,1]$} & $\mathcal{I}$ & {$[0,0]$} & {$[1,1]$} & {$[0,1]$} \\
\hline$[0,0][0,0]$ & {$[0,0]$} & {$[0,0]$} & & {$[0,0]$} & {$[1,1]$} & {$[1,1]$} & {$[1,1]$} \\
{$[1,1]$} & {$[0,0]$} & {$[1,1]$} & {$[0,1]$} & {$[1,1]$} & {$[0,0]$} & {$[1,1]$} & {$[0,1]$} \\
{$[0,1]$} & {$[0,0]$} & {$[0,1]$} & {$[0,0]$} & {$[0,1]$} & {$[0,1]$} & {$[1,1]$} & {$[1,1]$}
\end{tabular}

Then $\mathcal{I}=\mathcal{I}_{\mathcal{T}}$, the residuation principle is satisfied in this case, and as it is easily verified (e.g., by checking the truth tables), $\left(I\left(L_{2}\right), \leq_{t}, \mathcal{T}\right)$ is an MV-algebra.

We note, finally, that even if a Kleene negator exists on $\left(I(L), \leq_{t}\right)$, it might happen that there is no t-norm $\mathcal{T}$ on this triangle such that $\left(I(L), \leq_{t}, \mathcal{T}\right)$ is an MV-algebra. An example of this situation is the triangle $\mathcal{I}\left(\mathcal{L}_{3}\right)$ from Example 2. As we have shown (Example 4-b), there exists a Kleene-negator on $\left(I\left(L_{3}\right), \leq_{t}\right)$. Nevertheless, there is no t-norm on $\left(I\left(L_{3}\right), \leq_{t}\right)$ satisfying Conditions $(7)$ and $(8)$.

\section{Conclusion}

In this paper we have described an ongoing work that identifies bilattices, and in particular the constructs of bilattice-based squares and triangles, as appropriate structures for relating IVFSs and IFSs within one uniform and general framework. By not constraining ourselves to consistent elements only, a natural setting to represent and handle contradictions emerges.

We have shown that the definition and representation of suitable logical connectives within this setting can benefit a lot from bringing together results from both bilattice and $\mathcal{L}$-fuzzy set theory, and - moreover - it raises many non-trivial questions regarding the inter-relationships among the various alternatives. In a forthcoming paper we illustrate the application potential and the intuitive appeal of our framework in the context of preference modeling, showing that our approach clarifies and simplifies exiting works in this area (e.g., [17] and [25]).

\section{Acknowledgements}

This paper was prepared during the second author's visit to the Academic College of Tel-Aviv, sponsored by the National Science Foundation-Flanders. 


\section{References}

1. O. Arieli and A. Avron. Reasoning with logical bilattices. Journal of Logic, Language, and Information, 5(1):25-63, 1996.

2. O. Arieli and A. Avron. Bilattices and paraconsistency. In Frontiers of Paraconsistent Logic, pages 11-27. Research Studies Press, 2000.

3. O. Arieli, C. Cornelis, G. Deschrijver, and E. Kerre. Relating intuitionistic fuzzy sets and interval-valued fuzzy sets through bilattices. In Applied Computational Intelligence, pages 57-64. World Scientific, 2004.

4. K. T. Atanassov. Intuitionistic fuzzy sets, 1983. VII ITKR's Session, Sofia (deposed in Central Sci.-Technical Library of Bulg. Acad. of Sci., 1697/84) (in Bulgarian).

5. K. T. Atanassov. Remark on a property of the intuitionistic fuzzy interpretation triangle. Notes on Intuitionistic Fuzzy Sets, 8:8-37, 2002.

6. N. D. Belnap. How a computer should think. In G. Ryle, editor, Contemporary Aspects of Philosophy, pages 30-56. Oriel Press, 1977.

7. N. D. Belnap. A useful four-valued logic. In Modern Uses of Multiple-Valued Logic, pages 7-37. Reidel Publishing Company, 1977.

8. C. Chang. Algebraic analysis of many valued logics. Trans. AMS, 93:74-80, 1958.

9. C. Cornelis. Two-sidedness in the representation and processing of imprecise information, 2004. Ph. D. Thesis, Ghent University.

10. C. Cornelis, K. T. Atanassov, and E. Kerre. Intuitionistic fuzzy sets and intervalvalued fuzzy sets: a comparison. In Proc. EUSFLAT'03, pages 159-163, 2003.

11. C. Cornelis, G. Deschrijver, and E. Kerre. Implication in intuitionistic and intervalvalued fuzzy set theory: Construction, classification, application. International Journal of Approximate Reasoning, 35(1):55-95, 2004.

12. G. Deschrijver, C. Cornelis, and E. Kerre. Square and triangle: a comparison. In Proc. IPMU'04, pages 1389-1396, 2004.

13. G. Deschrijver and E. Kerre. On the relationship between some extensions of fuzzy set theory. Fuzzy Sets and Systems, 133(2):227-235, 2003.

14. M. Fitting. Personal communication.

15. M. Fitting. Bilattices and the semantics of logic programming. Journal of Logic Programming, 11(2):91-116, 1991.

16. M. Fitting. Kleene's logic, generalized. Logic and Computation, 1:797-810, 1992.

17. P. Fortemps and R. Słowiński. A graded quadrivalent logic for ordinal preference modelling. Fuzzy Optimization and Decision Making, 1:93-111, 2002.

18. G. Gargov. Knowledge, uncertainty and ignorance in logic: bilattices and beyond. Journal of Applied Non-Classical Logics, 9(2-3):195-283, 1999.

19. M. L. Ginsberg. Multi-valued logics: A uniform approach to reasoning in artificial intelligence. Computer Intelligence, 4:256-316, 1988.

20. J. Goguen. L-fuzzy sets. Journal Math. Anal. Appl., 18:145-174, 1967.

21. S. Jenei and B. De Baets. On the direct decomposability of t-norms onproduct lattices. Fuzzy Sets and Systems, 139(3):699-707, 2003.

22. B. Messing. Combining knowledge with many-valued logics. Data and Knowledge Engineering, 23:297-315, 1997.

23. R. Nelken and N. Francez. Bilattices and the semantics of natural language questions. Linguistic and Philosophy, 25(1):37-64, 2002.

24. G. Takeuti and S. Titani. Intuitionistic fuzzy logic and intuitionistic fuzzy sets theory. Journal of Symbolic Logic, 49:851-866, 1984.

25. A. Tsoukiàs and P. Vincke. Extended preference structures in mcda. In J. Clímaco, editor, Multi-criteria Analysis, pages 37-50. Springer-Verlag, 1997.

26. E. Turunen. Mathematics behind fuzzy logic. Advances in Soft Computing, 1999.

27. L. A. Zadeh. Fuzzy sets. Information and Control, 8:338-353, 1965. 